\title{
AFRONTAMIENTO DEL ESTRÉS ACADÉMICO Y AUTOEFICACIA EN ESTUDIANTES UNIVERSITARIOS: UN ENFOQUE BASADO EN PERFILES
}

\author{
Carlos Freire Rodríguez \\ Departamento de Psicología \\ Universidade da Coruña (España) \\ carlos.freire.rodriguez@udc.es \\ María del Mar Ferradás Canedo \\ Departamento de Psicología \\ Universidade da Coruña (España)
}

Recepción Artículo: 15 abril 2020

Admisión Evaluación: 17 abril 2020

Informe Evaluador 1: 17 abril 2020

Informe Evaluador 2: 119 abril 2020

Aprobación Publicación: 20 abril 2020

\section{RESUMEN}

Los estudios sobre estrés académico constatan el importante rol desempeñado por la autoeficacia y las estrategias de afrontamiento en la prevención de estados patológicos en los estudiantes. Sin embargo, hasta la fecha carecemos de evidencias respecto a la posible existencia de perfiles diferenciados de estudiantes en función de la forma en que conjugan diferentes estrategias de afrontamiento con sus expectativas de autoeficacia. Este planteamiento basado en la persona permitiría, por tanto, determinar qué estudiantes se encontrarían en una situación de mayor y menor vulnerabilidad ante el estrés. 1072 estudiantes de la Universidade da Coruña (España), con edades comprendidas entre los 18 y los 48 años $(M=21.09 ; D T=3.16)$ participaron en la investigación. 729 participantes (68\%) eran mujeres y 343 (32\%), hombres. Mediante un análisis de perfiles latentes (LPA) se identificaron tres perfiles de estudiantes: un grupo de afrontamiento adaptativo (alta autoeficacia y alta utilización de estrategias de reevaluación positiva, búsqueda de apoyo y planificación), un grupo de afrontamiento desadaptativo (baja autoeficacia y baja utilización de estrategias de reevaluación positiva, búsqueda de apoyo y planificación) y un grupo con elevada búsqueda de apoyo y baja autoeficacia combinada con baja reevaluación positiva y planificación. Los resultados de este estudio evidencian la existencia de diferentes tipologías de estudiantes en función de la forma en que conjugan su autoeficacia y sus estrategias de afrontamiento. Por tanto, estos hallazgos parecen refrendar la conveniencia de analizar el afrontamiento del estrés académico universitario desde la flexibilidad de estrategias de afrontamiento en combinación con las creencias de competencia autopercibida.

Palabras clave: estrés; afrontamiento; autoeficacia; perfiles; estudiantes universitarios

\section{ABSTRACT}

Coping with academic stress and self-efficacy in university students: a profile-based approach. Studies on academic stress confirm the important role played by self-efficacy and coping strategies 


\section{AFRONTAMIENTO DEL ESTRÉS ACADÉMICO Y AUTOEFICACIA EN ESTUDIANTES UNIVERSITARIOS: UN ENFOQUE BASADO EN PERFILES}

in the prevention of pathological conditions in students. However, to date we lack evidence regarding the possible existence of differentiated profiles of students depending on the way they combine different coping strategies with their self-efficacy expectations. This person-based approach would therefore allow to determine which students would be in a situation of greater and lesser vulnerability to stress. 1072 students from the University of Corunna (Spain), aged between 18 and 48 years $(M=21.09 ; S D=3.16)$ participated in the research. 729 participants (68\%) were women and 343 (32\%), men. Through a latent profile analysis (LPA), three student profiles were identified: an adaptive coping group (high self-efficacy and high use of positive reappraisal, support seeking, and planning), a maladaptive coping group (low self-efficacy and low use of positive reappraisal, seeking support, and planning), and a group with high seeking support combined with low self-efficacy, low positive reappraisal and low planning. The results of this study show the existence of different types of students depending on the way they combine their self-efficacy and their coping strategies. Therefore, these findings seem to endorse the desirability of analyzing coping with university academic stress from the flexibility of coping strategies in combination with the beliefs of self-perceived competence.

Keywords: stress; coping; self-efficacy; profiles; university students

\section{ANTECEDENTES}

Estudios recientes constatan un creciente empobrecimiento de la salud psicológica del alumnado universitario (Lipson, Lattie y Eisenberg, 2019), siendo el estrés una de las patologías más prevalentes en esta población (American College Health Association, 2018). En su día a día, los estudiantes deben hacer frente a múltiples y heterogéneas demandas (Beiter et al., 2015), entre las que se encontrarían algunas de tipo académico, como la adaptación a un nuevo entorno, la sobrecarga de trabajo o la evaluación, a las que se añadirían otras de tipo social (establecer nuevos vínculos, trabajar en equipo, vivir lejos del lugar de origen) y económico (recursos limitados, compatibilización de los estudios con un empleo remunerado). Estas demandas pueden repercutir negativamente sobre el estudiantado, tanto en su bienestar físico y psicológico (e.g., trastornos del sueño, ansiedad, irascibilidad, desregulación del apetito, consumo de sustancias adictivas) como en su rendimiento académico (Saklofske, Austin, Mastoras, Beaton y Osborne, 2012).

Si bien estas demandas constituyen una potencial amenaza, su grado de repercusión dependerá, en buena medida, de la respuesta que el estudiante proporcione en dichas situaciones. En efecto, en la actualidad existe un amplio acuerdo en la consideración del estrés como un proceso interactivo persona-ambiente, en virtud del cual los recursos bio-psico-sociales del individuo ejercen un papel central en la prevención del estrés. De entre los recursos psicológicos más ampliamente citados por la investigación sobresalen las estrategias de afrontamiento y las expectativas de autoeficacia.

Las estrategias de afrontamiento fueron definidas por Lazarus y Folkman (1986) como los "esfuerzos cognitivos y conductuales constantemente cambiantes que se desarrollan para manejar las demandas específicas externas y/0 internas que son evaluadas como excedentes o desbordantes de los recursos del individuo" (p. 164). Estas estrategias, por tanto, se erigen en verdaderas tentativas de eliminar 0, cuanto menos, reducir el impacto del estímulo estresor y, en consecuencia, prevenir la respuesta de estrés. Pese a que la funcionalidad de las estrategias de afrontamiento es altamente situacional, en general, se afirma que las estrategias de índole aproximativa son más adaptativas que las de carácter evasivo (Syed and Seiffge-Krenke, 2015). Las estrategias aproximativas son aquellas dirigidas a dar una respuesta activa al estresor, bien con objeto de modificar de forma directa el problema (control primario), bien con la pretensión de que este produzca un menor impacto emocional (control secundario). En esta categoría se encontrarían estrategias como la planificación, la búsqueda de apoyo, la reevaluación positiva de la situación o la aceptación de la misma. Por el contrario, las estrategias evasivas englobarían cogniciones y conductas desplegadas con afán de evitar la situación amenazante, entre las que podrían citarse la negación, la distracción o el pensamiento desiderativo.

Por lo que respecta a la autoeficacia, se trata de un constructo central en la Teoría social-cognitiva de Bandura (1997) que alude a la expectativa autorreferida relativa a la propia competencia para lograr con éxito un determi- 
nado estándar deseado. Esta expectativa puede variar en función de cada tarea específica, si bien ha sido conceptualizada también como una creencia generalizada en torno a la competencia percibida para hacer frente a una amplia gama de demandas. La autoeficacia ha recibido una considerable atención en contextos académicos, identificándose como uno de los determinantes más importantes de la conducta motivada del estudiante (Zumbrunn, Broda, Varier y Conklin, 2019). Además de su vertiente motivacional, la autoeficacia goza de un papel importante en los procesos de afrontamiento del estrés (Godoy-Izquierdo et al., 2008), influyendo decisivamente en la selección y ejecución del tipo de esfuerzos (aproximativos o evasivos) que el individuo pone en marcha para hacer frente a las potenciales amenazas.

Hasta la fecha, los estudios que han analizado el papel de la autoeficacia en el afrontamiento del estrés han adoptado un enfoque centrado en la variable. Esta perspectiva metodológica asume un planteamiento unívoco, según el cual los juicios percibidos en torno a las capacidades personales influirían en la elección de la estrategia concreta a utilizar para hacer frente a la situación. Como alternativa a esta perspectiva, un cuerpo creciente de trabajos aboga por analizar la flexibilidad del afrontamiento (e.g., Heffer y Willoughby, 2017), bajo la consideración de que algunas personas disponen de un espectro diverso de estrategias (i.e., perfiles de afrontamiento), de manera que muestran una elevada capacidad para simultanearlas (e.g., Schmidt, Nachtigall, Wuenthrich-Martone y Strauss, 2002). Por el contrario, aquellos individuos que exhiben una elevada inflexibilidad en sus mecanismos de afrontamiento tienden a recurrir sistemáticamente a la misma estrategia, 0 a un elenco reducido de ellas, con independencia de las características de la situación (Cheng y Cheung, 2005).

Si bien la investigación precedente ofrece evidencias acerca de la existencia de perfiles diferenciados de estudiantes en virtud del grado de flexibilidad en su afrontamiento, carecemos de precedentes que hayan tomado en consideración el papel de la autoeficacia en la conformación de estos perfiles. Desde esta consideración, el presente estudio pretende identificar las características de los perfiles de afrontamiento del estudiantado universitario en función de la forma en que conjugan las creencias de autoeficacia generalizadas con tres estrategias aproximativas de afrontamiento altamente adaptativas en el contexto académico (Skinner, Edge, Altman y Sherwood, 2003), como son la reevaluación positiva, la búsqueda de apoyo y la planificación. El carácter descriptivo exploratorio de este estudio no permite establecer hipótesis precisas respecto a los perfiles esperables, si bien resulta plausible considerar, al menos, la existencia de un perfil de estudiantes con altos niveles de autoeficacia y alta utilización de las tres estrategias, así como un perfil que conjugue una baja autoeficacia con una baja utilización de las tres estrategias.

\section{MÉTODO}

\section{Participantes}

En el estudio participaron 1072 estudiantes (729 mujeres y 343 hombres) de la Universidade da Coruña (España), seleccionados mediante un muestreo no probabilístico por conveniencia. Las edades de los participantes se encontraban en un rango de entre 18 y 48 años $(M=21.09 ; D T=3.16)$. Del total de la muestra, el 35.7\% ( $n=383$ ) de los participantes cursaban estudios afines a Ciencias de la Educación (Educación Infantil, Educación Primaria, Educación Física, Audición y Lenguaje, Educación Social Logopedia y Psicopedagogía); el 19\% ( $n=$ 203), estudios adscritos a Ciencias de la Salud (Fisioterapia, Enfermería y Ciencias de la Actividad Física y el Deporte); el 26\% ( $n=279)$ de los participantes estudiaban titulaciones de la rama técnica (Arquitectura, Arquitectura Técnica e Ingeniería de Caminos, Canales y Puertos); y el 19.3\% ( $n=207)$ cursaban estudios del ámbito jurídico-social (Derecho y Sociología).

\section{INSTRUMENTOS}

Estrategias aproximativas de afrontamiento del estrés. Las estrategias aproximativas de afrontamiento fueron evaluadas mediante la Escala de Afrontamiento del Cuestionario de Estrés Académico (Cabanach, Valle, Rodríguez, Piñeiro y Freire, 2010). El instrumento consta de 23 ítems que evalúan tres estrategias aproxi- 


\section{AFRONTAMIENTO DEL ESTRÉS ACADÉMICO Y AUTOEFICACIA EN ESTUDIANTES UNIVERSITARIOS: UN ENFOQUE BASADO EN PERFILES}

mativas de afrontamiento del estrés: reevaluación positiva (diez ítems, e.g., "Cuando me enfrento a una situación problemática, olvido los aspectos desagradables y resalto lo positivo"); búsqueda de apoyo (siete ítems, e.g., "Cuando me enfrento a una situación problemática, pido consejo a un familiar o a un amigo a quien aprecio"); y planificación (seis ítems, e.g., "Cuando me enfrento a una situación problemática mientras estoy preparando los exámenes, planifico detalladamente cómo estudiar el examen"). Las respuestas a los ítems se midieron una escala Likert de cinco puntos ( $1=$ Nunca $-5=$ Siempre). La consistencia interna del instrumento en el presente estudio fue de $\alpha=.86$ (reevaluación positiva), $\alpha=.90$ (búsqueda de apoyo) y $\alpha=.81$ (planificación).

Autoeficacia general. Se utilizó la validación española de la Escala de Autoeficacia General (Baessler y Schwarzer, 1996). La escala incluye diez ítems (e.g., "Tengo confianza en que podría manejar eficazmente acontecimientos inesperados"), a los que los participantes respondieron indicando una puntuación en una escala Likert con un rango entre 1 (Nunca) y 5 (Siempre). En el presente estudio, la consistencia interna del instrumento fue $\alpha=.91$.

\section{PROCEDIMIENTO}

La investigación fue desarrollada de acuerdo con los principios éticos que rigen las investigaciones con seres humanos, con consentimiento informado por escrito de los participantes, tal y como contempla la Declaración de Helsinki. Participaron en el estudio los estudiantes de aquellas titulaciones que voluntariamente aceptaron formar parte de la investigación. A los participantes se les solicitó su colaboración desinteresada, indicándoseles los objetivos de la investigación y garantizándoseles el anonimato y la confidencialidad de sus datos.

\section{ANÁLISIS DE DATOS}

La identificación de perfiles de afrontamiento y autoeficacia se llevó a cabo mediante un análisis de perfiles latentes (LPA), mediante el programa estadístico MPlus 7.11 (Muthén y Muthén, 1998-2012). De entre un conjunto finito de modelos, se determinó aquel que mostraba un mejor ajuste a los datos. Como parámetros de referencia para determinar el modelo óptimo se tuvieron en cuenta los siguientes: criterio de información de Akaike (AIC), criterio de información bayesiana de Schwarz (BIC), el BIC ajustado por el tamaño de la muestra (SSABIC), la prueba formal de la razón de máxima verosimilitud ajustada de Lo, Mendell y Rubin (2001) -LMRT-, la prueba paramétrica de la razón de verosimilitud de reemplazamiento (PBLRT) y el tamaño de la muestra para cada subgrupo. Los índices AIC, BIC y SSA-BIC tienen un carácter descriptivo, indicando los valores más bajos un mejor ajuste del modelo, si bien son los índices LMRT y PBLRT Ios que permiten tomar una decisión final. El valor $p$ asociado con el LMRT y el PBLRT indica si la solución con más $(p<.05)$ o menos clases $(p>$.05) es la que mejor se ajusta a los datos. Asimismo, se tomó como criterio de exclusión la existencia de clases espurias ( $n \leq 5 \%$ de la muestra), condición indicativa de una excesiva extracción de perfiles (Hipp y Bauer, 2006).

Una vez seleccionado el modelo óptimo con base en los criterios anteriormente citados, se procedió a determinar su precisión clasificatoria tomando como referencia el cálculo de las probabilidades a posteriori y el estadístico de entropía. Los valores de entropía oscilan entre cero y uno, indicando los valores más próximos a la unidad una mayor precisión clasificatoria. Otro criterio empleado para evaluar la validez del modelo seleccionado fue la realización de un ANOVA, para analizar las diferencias entre clases en las tres variables criterio (reevaluación positiva, búsqueda de apoyo y planificación). La existencia de diferencias estadísticamente significativas en las cuatro variables indicaría que las clases latentes sugeridas por el modelo seleccionado son distintas entre sí. 


\section{RESULTADOS}

\section{Análisis preliminares}

La Tabla 1 resume los estadísticos descriptivos y los índices de correlación entre las variables autoeficacia, reevaluación positiva, búsqueda de apoyo y planificación. Los valores de asimetría y curtosis son indicativos de que los datos se ajustan a una distribución normal (Finney y DiStefano, 2006). En lo que respecta a las correlaciones (Pearson) entre las variables, todas ellas son positivas y estadísticamente significativas $(p<.001)$.

Tabla 1

Medias, desviaciones típicas, asimetría, curtosis y matriz de correlaciones

\begin{tabular}{|c|c|c|c|c|}
\hline & 1 & 2 & 3 & 4 \\
\hline 1. AUTOE & - & & & \\
\hline 2. RE POS & $.63^{* *}$ & - & & \\
\hline 3. BU_APO & $.21 * *$ & $.22 * *$ & - & \\
\hline 4. PLANNIF & $.45^{* *}$ & $.55^{* *}$ & $.30 * *$ & - \\
\hline$M$ & 3.34 & 3.01 & 3.44 & 3.05 \\
\hline$D T$ & 0.68 & 0.71 & 0.87 & 0.74 \\
\hline Asimetría & 0.03 & 0.05 & -0.15 & 0.07 \\
\hline Curtosis & -0.44 & -0.46 & -0.79 & -0.44 \\
\hline
\end{tabular}

Nota. AUTOE $=$ Autoeficacia; RE_POS $=$ Reevaluación Positiva; BU_APO $=$ Búsqueda de Apoyo; PLANIF = Planificación.

$* * p<0.01$

\section{Identificación de los perfiles de autoeficacia y estrategias de afrontamiento}

Varios modelos de perfiles latentes han sido analizados con base en los datos de la muestra (modelos de dos a cuatro clases; véase Tabla 2). Se ha detenido el ajuste de modelos en cuatro clases, al obtenerse un LMRT no significativo, indicativo de que este modelo no presenta un ajuste significativamente mejor que el modelo precedente. Asimismo, al comparar el modelo de tres clases con el de dos clases se observaron valores AIC, BIC y SSA-BIC inferiores, lo que sugiere un mejor ajuste del modelo de tres clases.

Tabla 2

Estadísticos para la identificación del ajuste de modelos de clases latentes y precisión clasificatoria

\begin{tabular}{lcccc}
\hline & 1 & 2 & 3 & 4 \\
\hline 1. AUTOE & - & & & \\
2. RE_POS & $.63^{* *}$ & - & & \\
3. BU_APO & $.21^{* *}$ & $.22^{* *}$ & - & \\
4. PLANIF & $.45^{* *}$ & $.55^{* *}$ & $.30^{* *}$ & - \\
& & & & \\
$M$ & 3.34 & 3.01 & 3.44 & 3.05 \\
DT & 0.68 & 0.71 & 0.87 & 0.74 \\
Asimetría & 0.03 & 0.05 & -0.15 & 0.07 \\
Curtosis & -0.44 & -0.46 & -0.79 & -0.44 \\
\hline
\end{tabular}

Nota . AUTOE $=$ Autoeficacia; RE_POS $=$ Reevaluación Positiva; BU_APO = Búsqueda de Apoyo; PLANIF = Planificación.

$* * p<0.01$ 


\section{AFRONTAMIENTO DEL ESTRÉS ACADÉMICO Y AUTOEFICACIA EN ESTUDIANTES UNIVERSITARIOS: UN ENFOQUE BASADO EN PERFILES}

La Tabla 3 informa de la precisión clasificatoria del modelo de cuatro clases, así como del número de participantes, que integran cada clase del modelo seleccionado, tanto en términos absolutos $(n)$ como porcentuales (\%). Los promedios asociados a los grupos a los que los participantes fueron asignados se presentan en la diagonal principal de la tabla, en negrita. Se observa en los tres grupos un coeficiente de clasificación superior al $80 \%$. En conjunto, estos datos indican que el modelo de tres clases presenta una adecuada precisión clasificatoria. Asimismo, el valor del estadístico de entropía de este modelo (.690) (Tabla 2) es aceptable (Nylund, Asparouhov y Muthén, 2007).

Tabla 3

Caracterización de las clases latentes y precisión de la clasificación de los individuos en cada clase

\begin{tabular}{cccccc}
\hline & 1 & 2 & 3 & $n$ & $\%$ \\
\cline { 2 - 6 } Clase 1 & $\mathbf{. 8 6 3}$ & .000 & .137 & 237 & 22.11 \\
Clase 2 & .000 & $\mathbf{. 8 8 8}$ & .112 & 302 & 28.17 \\
Clase 3 & .086 & .081 & $\mathbf{. 8 3 3}$ & 533 & 49.72 \\
\hline
\end{tabular}

Nota. Los coeficientes asignados a los grupos a los que los sujetos han sido asignados se muestran en negrita.

Como criterio adicional para determinar la idoneidad del modelo de tres clases, los resultados del MANOVA evidenciaron diferencias estadísticamente significativas entre las tres clases en las cuatro variables criterio: autoeficacia $\left(F(2,1069)=632.524 ; p<.001 ; p^{2}=.542\right)$, reevaluación positiva $\left(F(2,1069)=1319.449 ; p<.001 ; \eta_{p}^{2}\right.$ $=.712)$, búsqueda de apoyo $\left(F(2,1069)=71.138 ; p<.001 ; \eta_{p}{ }^{2}=.117\right)$, planificación $(F(2,1069)=389.071 ; p$ $\left.<.001 ; \eta_{p}^{2}=.421\right)$. El tamaño del efecto fue grande en todos los casos.

\section{DESCRIPCIÓN DE LOS PERFILES DE AUTOEFICACIA Y ESTRATEGIAS DE AFRONTAMIENTO}

Las puntuaciones medias (directas y estandarizadas) de los sujetos pertenecientes a cada una de las clases latentes (perfiles de autoeficacia y estrategias de afrontamiento) del modelo seleccionado se muestran en la Tabla 4. Asimismo, en la Figura 1 se representan gráficamente estos perfiles. 
Tabla 4

Descripción de los perfiles (medias, desviaciones estándar e intervalos de confianza)

\begin{tabular}{lcccc} 
& \multicolumn{4}{c}{ Intervalos de confianza } \\
\hline & $M$ & $D E$ & Inferior 5\% & Superior 5\% \\
\hline$B A \_B E A(n=237)$ & & & & \\
Autoeficacia & $2.64(-1.12)$ & 0.07 & 2.52 & 2.75 \\
Reevaluación positiva & $2.21(-1.22)$ & 0.07 & 2.09 & 2.33 \\
Búsqueda de apoyo & $2.95(-0.63)$ & 0.10 & 2.79 & 3.10 \\
Planificación & $2.39(-0.93)$ & 0.07 & 2.28 & 2.51 \\
$A A_{-} A E A(n=302)$ & & & & \\
Autoeficacia & $3.97(0.97)$ & 0.06 & 3.87 & 4.06 \\
Reevaluación positiva & $3.79(1.16)$ & 0.06 & 3.68 & 3.89 \\
Búsqueda de apoyo & $3.69(028)$ & 0.06 & 3.60 & 3.78 \\
Planificación & $3.67(0.89)$ & 0.06 & 3.57 & 3.77 \\
$B A \_E S(n=533)$ & & & & \\
Autoeficacia & $3.30(-0.49)$ & 0.07 & 3.18 & 3.42 \\
Reevaluación positiva & $2.94(-0.12)$ & 0.08 & 2.80 & 3.07 \\
Búsqueda de apoyo & $3.53(0.12)$ & 0.06 & 3.43 & 3.64 \\
Planificación & $2.99(-0.09)$ & 0.07 & 2.87 & 3.11 \\
\hline
\end{tabular}

Nota. BA_BEA: perfil de baja autoeficacia y bajas estrategias aproximativas; AA_AEA: perfil de alta autoeficacia y altas estrategias aproximativas; BA_ES: perfil de baja autoeficacia y predominio de estrategias de carácter social. Las puntuaciones estandarizadas (z) aparecen entre paréntesis.

El primer grupo ( $n=237 ; 22.11 \%)$ está representado por estudiantes que conjugan bajas puntuaciones en autoeficacia general con bajas puntuaciones en las tres estrategias aproximativas de afrontamiento (perfil BA_BEA). Por tanto, se trataría de estudiantes con bajas creencias autorreferidas de competencia personal así como con una escasa flexibilidad en la adopción de estrategias enfocadas a la gestión de los problemas. El segundo grupo ( $n=302 ; 28.17 \%$ ) se caracteriza por el patrón opuesto, esto es, altos niveles de autoeficacia y alta utilización de las estrategias de búsqueda de apoyo, reevaluación positiva y planificación, en especial, de estas dos últimas (perfil AA_AEA). Finalmente, el tercer grupo de estudiantes ( $n=533 ; 49.72 \%)$ muestran puntuaciones moderadamente bajas en autoeficacia, así como en las estrategias de reevaluación positiva y planificación. Sin embargo, evidencian también puntuaciones moderadamente elevadas en la utilización de la estrategia de búsqueda de apoyo. Dado el carácter eminentemente social de esta estrategia (a diferencia de las otras dos, cuyas características son más cognitivas), hemos denominado a este grupo perfil de estudiantes con baja autoeficacia y predominio de utilización de estrategias de carácter social (perfil BA_ES). 


\section{AFRONTAMIENTO DEL ESTRÉS ACADÉMICO Y AUTOEFICACIA EN ESTUDIANTES UNIVERSITARIOS: UN ENFOQUE BASADO EN PERFILES}

Figura 1. Representación gráfica de los perfiles de autoeficacia y afrontamiento aproximativo

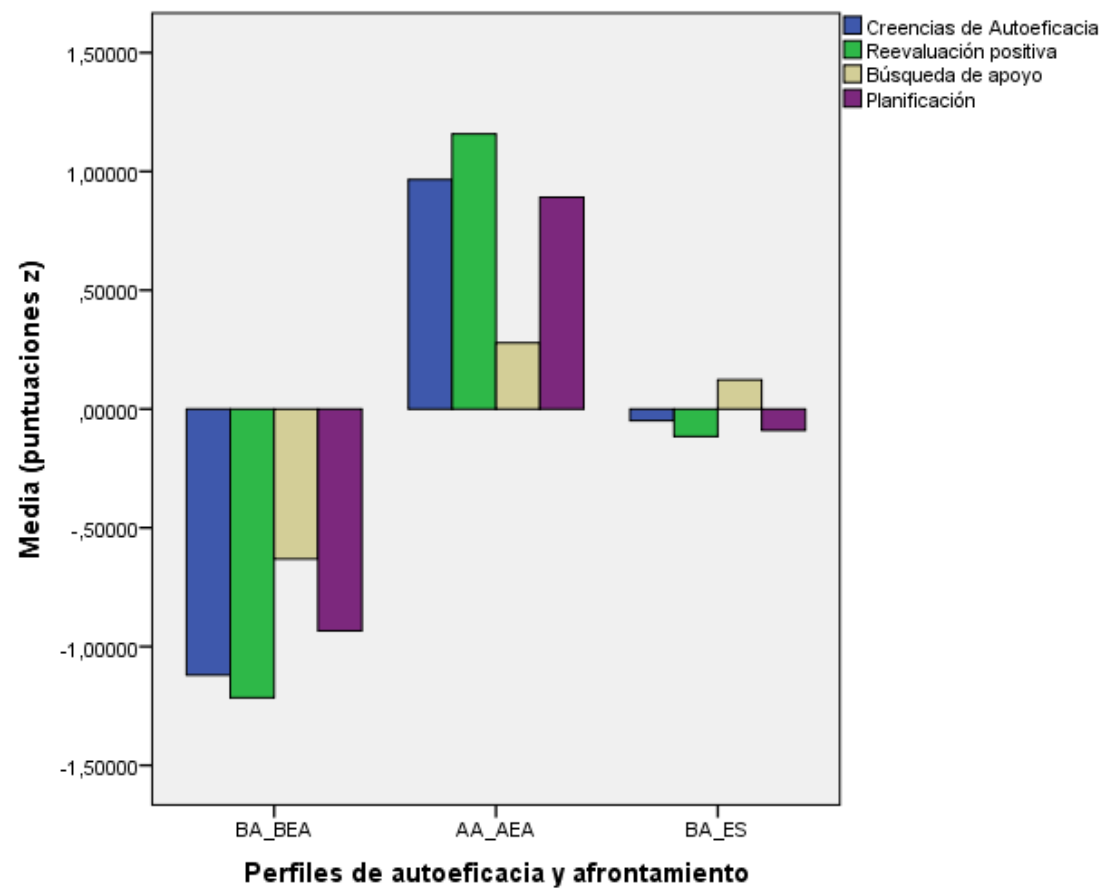

Nota. BA_BEA: perfil de baja autoeficacia y bajas estrategias aproximativas; AA_AEA: perfil de alta autoeficacia y altas estrategias aproximativas; BA_ES: perfil de baja autoeficacia y predominio de estrategias de carácter social.

\section{DISCUSIÓN}

El presente trabajo se enmarca dentro de la línea creciente de investigaciones que, en consonancia con los planteamientos de la Psicología Positiva (e.g., Seligman, 2011), enfatizan la importancia de los recursos psicológicos personales en la prevención y reducción de estados psicopatológicos. La principal aportación de este estudio ha sido la identificación de perfiles de estudiantes diferenciados entre sí en cuanto a la forma en que conjugan sus expectativas generalizadas de autoeficacia con diversas estrategias aproximativas de afrontamiento a la hora de hacer frente al estrés.

En este sentido, nuestros hallazgos sugieren la existencia de un perfil de estudiantes (perfil AA_AEA) que aunarían unas altas creencias de autoeficacia con una elevada flexibilidad para simultanear tres estrategias altamente funcionales en el ámbito académico (Skinner et al., 2003), como la reevaluación positiva de los problemas, la planificación y la búsqueda de apoyo. Se trataría, por tanto, de estudiantes que confiarían en su propia competencia para hacer frente a las demandas a las que deben hacer frente en su día a día universitario, y desplegarían, para ello, un amplio elenco de estrategias, que les posibilitaría resignificar los problemas, extrayendo los aspectos positivos de estos, planificar detenidamente la forma de abordarlos y buscar la ayuda de otras personas con fines instrumentales y emocionales. Asumiendo que los perfiles de afrontamiento que conjugan diversas estrategias aproximativas, en comparación con los perfiles menos flexibles en el afrontamiento aproximativo, son más resilientes al estrés (Lee, Seo, Lee, Park y Lee, 2016) y al burnout (Martinent y Decret, 2015), y más proclives 
a experimentar bienestar psicológico (Freire, Ferradás, Núñez y Valle, 2018), podría concluirse que el perfil AA_AEA resulta altamente funcional para la prevención del estrés universitario.

En el extremo opuesto se encontraría el perfil BA_BEA. Los estudiantes encuadrados en este grupo evidencian unas bajas expectativas de autoeficacia, así como una escasa utilización de las tres estrategias aproximativas analizadas. Estos estudiantes poseen una escasa confianza autopercibida para hacer frente a las demandas de la etapa universitaria, lo que posiblemente explicaría, cuando menos parcialmente, su escaso afán por desplegar estrategias dirigidas a darles una respuesta activa. Por consiguiente, y en consonancia con la mayor vulnerabilidad al estrés evidenciada por los perfiles con baja flexibilidad en la utilización de estrategias aproximativas (González-Cabanach, Souto-Gestal, González-Doniz y Franco, 2018), el perfil BA_BEA resultaría altamente disfuncional.

Finalmente, se identificó un tercer grupo de estudiantes (perfil BA_ES) con unos niveles de autoeficacia moderadamente bajos que, en lo tocante a sus estrategias de afrontamiento, muestran un predominio por aproximarse a las demandas universitarias buscando apoyo, instrumental y emocional, en otras personas. Sin embargo, no recurrirían a la adopción de estrategias aproximativas de índole cognitiva (i.e., planificación y reevaluación positiva). De acuerdo con esta caracterización, es posible que los estudiantes encuadrados en este grupo se perciban poco competentes para dar respuesta por sí mismos a los estresores académicos y extraacadémicos, evidenciando una excesiva dependencia hacia su círculo social significativo.

En conjunto, Ios resultados del presente estudio permiten identificar a aquel estudiantado que, con base en sus características de autoeficacia general y utilización de estrategias aproximativas de afrontamiento, se encuentran en una mayor (perfil BA_BEA) y menor (perfil AA_AEA) vulnerabilidad a desarrollar estrés durante la etapa universitaria. No obstante, estos hallazgos deben asumirse con cautela, habida cuenta de las limitaciones del estudio efectuado. En primer lugar, el procedimiento de muestreo empleado dificulta la generalización de los resultados a la población universitaria en su conjunto. En segundo lugar, la utilización de procedimientos de autoinforme como medio de recogida de datos puede suponer un sesgo a la hora de obtener información veraz. Asimismo, no es descartable que los estudiantes hagan uso de otras estrategias de afrontamiento, tanto aproximativas como evasivas, no contempladas en este estudio, lo que podría condicionar la identificación de nuevos perfiles.

\section{REFERENCIAS BIBLIOGRÁFICAS}

American College Health Association (2018). National College Health Assessment II: Reference Group Executive Summary Fall 2018. Hanover, MD: American College Health Association.

Baessler, J., and Schwarzer, R. (1996). Evaluación de la autoeficacia: adaptación española de la escala de autoeficacia general. Ansiedad y Estrés 2, 1-8.

Bandura, A. (1997). Self-efficacy: The exercise of control. New York, NY: Freeman.

Beiter, R., Nash, R., McCrady, M., Rhoades, D., Linscomb, M., Clarahan, M., \& Sammut, S. (2015). The prevalence and correlates of depression, anxiety, and stress in a sample of college students. Journal of Affective Disorders, 173, 90-96. doi:10.1016/j.jad.2014.10.054

Cabanach, R. G., Valle, A., Rodríguez, S., Piñeiro, I., \& Freire, C. (2010). Escala de Afrontamiento del Estrés Académico (A-CEA). Revista Iberoamericana de Psicología y Salud 1, 51-64.

Cheng, C., \& Cheung, M. W. L. (2005). Cognitive processes underlying coping flexibility: differentiation and integration. Journal of Personality, 73(4), 859-886. doi:10.1111/j.1467-6494.2005.00331.x

Freire, C., Ferradás, M. M., Núñez, J. C., \& Valle, A. (2018). Coping flexibility and eudaimonic well-being in university students. Scandinavian Journal of Psychology, 59(4), 433-442. doi:10.1111/sjop.12458

Godoy-Izquierdo, D., Godoy-García, J. F., López-Chicheri, I., Martínez, A., Gutiérrez, S., \& Vázquez, L. (2008). Propiedades psicométricas de la Escala de Autoeficacia para el Afrontamiento del Estrés (EAEAE). Psicothema, 20(1), 155-165. 


\section{AFRONTAMIENTO DEL ESTRÉS ACADÉMICO Y AUTOEFICACIA EN ESTUDIANTES UNIVERSITARIOS: UN ENFOQUE BASADO EN PERFILES}

González-Cabanach, R., Souto-Gestal, A., González-Doniz, L., \& Franco, V. (2018). Perfiles de afrontamiento y estrés académico en estudiantes universitarios. Revista de Investigación en Educación, 36, 421-433. doi:10.6018/rie.36.2.290901

Heffer, T., \& Willoughby, T. (2017). A count of coping strategies: A longitudinal study investigating an alternative method to understanding coping and adjustment. PLOS One, 12(10):e0186057. doi:10.1371/journal.pone.0186057

Hipp, J. R., \& Bauer, D. J. (2006). Local solutions in the estimation of growth mixture models. Psychological Methods 11, 36-53. doi: 10.1037/1082-989X.11.1.36

Lazarus, R. S., \& Folkman, S. (1986). Estrés y procesos cognitivos. Barcelona: Martínez Roca.

Lee, J. H., Seo, M., Lee, M., Park, S. Y., \& Lee, J. H. (2016). Profiles of coping strategies in resilient adolescents. Psychological Reports, 120(1), 49-69. doi:10.1177/0033294116677947

Lipson, S. K., Lattie, E. G., \& Eisenberg, D. (2019). Increased rates of mental health service utilization by U.S. college students: 10-year population-level trends (2007-2017). Psychiatric Services, 70(1), 60-63. doi:10.1176/appi.ps.201800332

Lo, Y., Mendell, N. R., \& Rubin, D. B. (2001). Testing the number of components in a normal mixture. Biometrika $88,767-778$.

Martinent, G., \& Decret, J. C. (2015). Coping profiles of young athletes in their everyday life: A three-wave twomonth study. European Journal of Sport Science, 15(8), 736-747. doi:10.1080/17461391.2015.1051131

Muthén, L. K., \& Muthén, B. 0. (1998-2012). Mplus User's Guide, 6th ed. Los Angeles, CA: Muthén \& Muthén.

Saklofske, D. H., Austin, E. J., Mastoras, S. M., Beaton, L., \& Osborne, S. E. (2012). Relationships of personality, affect, emotional intelligence and coping with Student stress and academic success: Different patterns of association for stress and success. Learning and Individual Differences, 22, 251-257. doi:10.1016/j.lindif.2011.02.010

Schmidt, S., Nachtigall, C., Wuenthrich-Martone, 0., \& Strauss, B. (2002). Attachment and coping with chronic disease. Journal of Psychosomatic Research, 53(3), 763-773. doi:10.1016/S0022-3999(02)00335-5

Seligman, M. E. P. (2011). La vida que florece. Barcelona: Ediciones B.

Skinner, E. A., Edge, K., Altman, J., and Sherwood, H. (2003). Searching for the structure of coping: A review and critique of category systems for classifying ways of coping. Psychological Bulletin, 129, 216-269. doi:10.1037/0033-2909.129.2.216

Syed, M., \& Seiffge-Krenke, I. (2015). Change in ego development, coping, and symptomatology from adolescence to emerging adulthood. Journal of Applied and Developmental Psychology, 41, 110-119. doi:10.1016/j.appdev.2015.09.003

Zumbrunn, S., Broda, M., Varier, S., \& Conklin, S. (2019). Examining the multidimensional role of self-efficacy for writing on student writing self-regulation and grades in elementary and high school. British Journal of Educational Psychology doi:10.1111/bjep.12315 